\title{
Effect of Partially Polarized Amplified Spontaneous Emission Noise on $Q$-Factor Estimation Using Optical Signal-to-Noise Ratio
}

\author{
J. H. Lee, D. M. Yeo, and Y. C. Chung
}

\begin{abstract}
We report on the effect of the partially polarized amplified spontaneous emission noise on the $Q$-factor estimation by using optical signal-to-noise ratio. The result shows that this effect is negligible even in a long-distance transmission system (number of spans: <40) as long as the polarization-dependent loss per span is smaller than $0.2 \mathrm{~dB}$.
\end{abstract}

Index Terms-Amplified spontaneous emission (ASE) noise, degree of polarization (DOP), polarization-dependent loss (PDL), statistics.

\section{INTRODUCTION}

$\mathbf{O}$ PTICAL signal-to-noise ratio (OSNR) has been widely used to estimate the performance of wavelength-divisionmultiplexed (WDM) system. However, it has been recently reported that such estimation could become inaccurate if the amplified spontaneous emission (ASE) noise is partially polarized due to polarization-dependent loss (PDL) [1], [2]. In fact, it has long been observed in a recirculating loop that the ASE noise could be polarized significantly by PDL when the polarization scrambling is not used [3]. To solve this problem, it would be necessary to identify the degree of polarization (DOP) of ASE noise and the relative polarization angle between the optical signal and the partially polarized ASE noise [1]. However, due to their random nature, it is difficult to measure these parameters accurately and use them for the correction of the estimated performance. Thus, it would be helpful if we identify the conditions under which the system's performance could still be estimated by using OSNR without considering these parameters. In this letter, we investigated such conditions by calculating the probability of estimation errors caused by the partially polarized ASE noise. For this purpose, we analyzed the probability density function (pdf) of the DOP of ASE noise and verified it experimentally. The results show that, in most cases, the $Q$-factor can be estimated by OSNR without considering DOP of ASE noise. For example, the probability that the error in the estimated $Q$-factor by using OSNR becomes greater than $1 \mathrm{~dB}$ is smaller than $10^{-5}$ in a 30 -span transmission system as long as the PDL/span is smaller than $0.2 \mathrm{~dB}$.

\section{THEORY}

To analyze the DOP statistics of ASE noise, we considered a long-distance transmission link consisted of a number of am-

Manuscript received August 29, 2005; revised November 14, 2005.

The authors are with the Department of Electrical Engineering, Korea Advanced Institute of Science and Technology, Daejeon 305-701, Korea (e-mail: ychung@ee.kaist.ac.kr).

Digital Object Identifier 10.1109/LPT.2005.863178

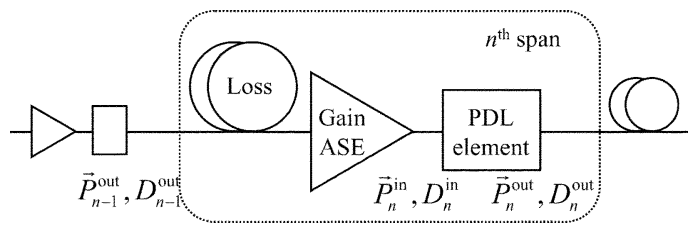

Fig. 1. Schematic diagram of the transmission link with distributed PDL elements. ( $\vec{P}_{n}^{\text {in }}$ and $\vec{P}_{n}^{\text {out }}$ are Stokes vectors of the ASE noise at the input and output of the $n$th PDL element, respectively. $D_{n}^{\text {in }}$ and $D_{n}^{\text {out }}$ are the powers of the unpolarized portion of ASE noise at the input and output of the $n$th PDL element, respectively.)

plifier spans, as shown in Fig. 1. Each span was composed of a transmission fiber, an optical amplifier, and a PDL element. For simplicity, we assumed that the total PDL within a span, caused by the transmission fiber and various optical components including optical amplifier, could be represented solely by the PDL element [4]. We also assumed that the birefringence in the transmission fiber was large enough to randomize the polarization states of the optical signals between the PDL elements [4]. However, polarization-mode dispersion (PMD) caused by birefringence should be too small to alter the DOP of the partially polarized ASE noise. This assumption is justifiable since the optical bandwidth of ASE noise used for the evaluation of OSNR is very narrow (i.e., similar to the signal's bandwidth). The averaged amplifier gain, over all the WDM channels, was assumed to be identical to the span loss [4]. However, the number of channels should be sufficiently large to justify this assumption. Otherwise, the amplifier gain could become dependent on the polarization states of input signals.

The Stokes vector of the ASE noise at the input of the $n$th PDL element can be expressed as $\vec{P}_{n}^{\text {in }}=R_{n} \vec{P}_{n-1}^{\text {out }}$, where $R_{n}$ is a $3 \times 3$ unitary matrix representing the polarization rotation caused by the birefringence of the transmission fiber in the $n$th span, and $\vec{P}_{n-1}^{\text {out }}$ is the Stokes vector of the ASE noise at the output of the $(n-1)$ th PDL element. Thus, the magnitudes of $\vec{P}_{n}^{\text {in }}$ and $\vec{P}_{n-1}^{\text {out }}$ should be identical (i.e., $\left.\left|\vec{P}_{n}^{\text {in }}\right|=\left|\vec{P}_{n-1}^{\text {out }}\right|\right)$. We assume that the power of ASE noise added by each amplifier is equal to one. Thus, the power of the unpolarized portion of ASE noise at the input of the $n$th PDL element can be expressed as $D_{n}^{\text {in }}=D_{n-1}^{\text {out }}+1$, where $D_{n-1}^{\text {out }}$ is the power of the unpolarized portion of ASE noise at the output of the $(n-1)^{\text {th }}$ PDL element. $\vec{P}_{n}^{\text {out }}$ and $D_{n}^{\text {out }}$ can be expressed as

$$
\begin{aligned}
\vec{P}_{n}^{\text {out }}= & T_{n} \vec{P}_{n}^{\text {in }}\left(1+\vec{\Gamma}_{n} \cdot \hat{p}_{n}^{\text {in }}\right)+D_{n}^{\text {in }} \vec{\Gamma}_{n} \\
D_{n}^{\text {out }}= & \left(1-\left|\vec{\Gamma}_{n}\right|\right) D_{n}^{\text {in }} \\
& +\left\{\left|\vec{P}_{n}^{\text {in }}\right|\left|1+\vec{\Gamma}_{n} \cdot \hat{p}_{n}^{\text {in }}\right|+D_{n}^{\text {in }}\left|\vec{\Gamma}_{n}\right|-\left|\vec{P}_{n}^{\text {out }}\right|\right\}
\end{aligned}
$$


where $T_{n}$ is a $3 \times 3$ matrix representing the polarization rotation caused by $n$th PDL element. The power variation caused by PDL is considered separately in the factor $\left(1+\vec{\Gamma}_{n} \cdot \hat{p}_{n}^{\text {in }}\right)$, where $\vec{\Gamma}_{n} \cdot \hat{p}_{n}^{\text {in }}$ represents the inner product of $\vec{\Gamma}_{n}$ and $\hat{p}_{n}^{\text {in }}, \vec{\Gamma}_{n}$ is the PDL vector of the $n$th PDL element, and $\hat{p}_{n}^{\text {in }}$ is the normalized Stokes vector of $\vec{P}_{n}^{\text {in }}$ (i.e., $\hat{p}_{n}^{\text {in }}=\vec{P}_{n}^{\text {in }} /\left|\vec{P}_{n}^{\text {in }}\right|$ ) [5]. The magnitude of the PDL vector $|\vec{\Gamma}|$ is defined as $\left(1-10^{- \text {pdl_dB } / 10}\right) /(1+$ $\left.10^{- \text {pdl_dB } / 10}\right)$, where pdl_dB represents PDL of the PDL element in decibels.

For small PDL (i.e., $|\vec{\Gamma}| \ll 1$ ), the total amount of ASE noise in the $n$th span can be approximated as $\left|\vec{P}_{n}^{\text {out }}\right|+D_{n}^{\text {out }} \cong n$. Thus, DOP of the ASE noise in the $n$th span can be expressed as

$$
\operatorname{DOP}_{n}=\frac{\left|\vec{P}_{n}^{\text {out }}\right|}{\left(\left|\vec{P}_{n}^{\text {out }}\right|+D_{n}^{\text {out }}\right)} \cong \frac{\left|\vec{P}_{n}^{\text {out }}\right|}{n} .
$$

This equation indicates that $\mathrm{DOP}_{n}$ is linearly proportional to $\left|\vec{P}_{n}^{\text {out }}\right|$. Thus, we can obtain the pdf of $\mathrm{DOP}_{n}$ directly from the pdf of $\left|\vec{P}_{n}^{\text {out }}\right|$. We first expand $\vec{P}_{n}^{\text {out }}$ in (1) into $n$ terms as

$$
\vec{P}_{n}^{\text {out }}=\vec{W}_{1}+\vec{W}_{2}+\cdots+\vec{W}_{n}
$$

where $\vec{W}_{k}=\hat{w}_{k}\left|\vec{W}_{k}\right|$, and

$$
\begin{aligned}
& \hat{w}_{1}=\frac{\vec{\Gamma}_{n}}{\left|\vec{\Gamma}_{n}\right|} \\
& \hat{w}_{k}=\frac{\left(\prod_{i=0}^{k-2} T_{n-i} R_{n-i}\right) \vec{\Gamma}_{n-k+1}}{\left|\vec{\Gamma}_{n-k+1}\right|}, \quad \text { for } k \geq 2
\end{aligned}
$$

$$
\begin{aligned}
\left|\vec{W}_{1}\right|= & D_{n}^{\text {in }}\left|\vec{\Gamma}_{n}\right|, \\
\left|\vec{W}_{k}\right|= & \left(\prod_{i=0}^{k-2}\left(1+\vec{\Gamma}_{n-i} \cdot \hat{p}_{n-i}^{\text {in }}\right)\right) \\
& \times D_{n-k+1}^{\text {in }}\left|\vec{\Gamma}_{n-k+1}\right|, \quad \text { for } k \geq 2 .
\end{aligned}
$$

In (5), $\hat{w}_{1}, \hat{w}_{2}, \ldots, \hat{w}_{n}$ are randomly oriented unit vectors since $R_{1}, R_{2}, \ldots, R_{n}$ are independent from each other and $\vec{\Gamma}_{1}, \vec{\Gamma}_{2}, \ldots, \vec{\Gamma}_{n}$ have random orientations. Thus, $\vec{P}_{n}^{\text {out }}$ can be regarded a sum of the randomly oriented three-dimensional vectors. It is well known from the PMD theory that the magnitude of such vector (i.e., $\left|\vec{P}_{n}^{\text {out }}\right|$ ) has a Maxwellian for large $n$ [6]. Thus, $\mathrm{DOP}_{n}$ should also have a Maxwellian for large $n$. As a result, the distribution of $\mathrm{DOP}_{n}$ can be expressed as

$$
p\left(\mathrm{DOP}_{n}\right)=\frac{32 \mathrm{DOP}_{n}^{2}}{\pi^{2}\left\langle\mathrm{DOP}_{n}\right\rangle^{3}} \exp \left(-\frac{1}{\pi}\left(\frac{2 \mathrm{DOP}_{n}}{\left\langle\mathrm{DOP}_{n}\right\rangle}\right)^{2}\right)
$$

where $\left\langle\mathrm{DOP}_{n}\right\rangle$ is the average DOP of the ASE noise in the $n$th span given by

$$
\begin{aligned}
\left\langle\mathrm{DOP}_{n}\right\rangle^{2}=(1 & \left.+\frac{2}{3} \Gamma^{2}\right)\left(1-\frac{1}{n}\right)^{2}\left\langle\mathrm{DOP}_{n-1}\right\rangle^{2} \\
& -\frac{16}{9 \pi}\left(1-\frac{1}{n}\right) \Gamma^{2}\left\langle\mathrm{DOP}_{n-1}\right\rangle+\frac{8}{3 \pi} \Gamma^{2}
\end{aligned}
$$

for $n \geq 2$ and $\left\langle\mathrm{DOP}_{1}\right\rangle=\Gamma$. In (8), we assume that all the PDL elements have the same PDL (i.e., $\left|\vec{\Gamma}_{n}\right|=\Gamma$ for all $n$ ).

When the ASE noise is partially polarized due to PDL, the $Q$-factor can be described as

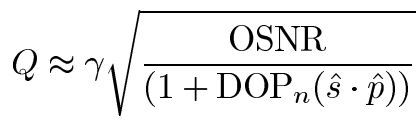

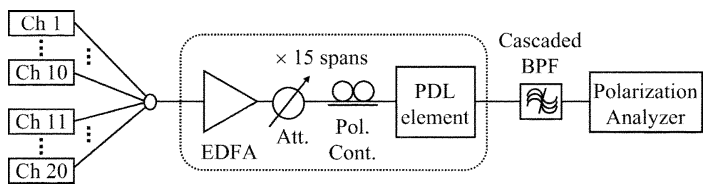

Fig. 2. Experimental setup to measure the DOP statistics of ASE noise (Att.: optical attenuator. Pol. Cont.: polarization controller. BPF: bandpass filter.)

for large OSNR (i.e., OSNR $\gg 10 \mathrm{~dB}$ ), where $\gamma$ is a constant determined by the modulation format and receiver characteristics, and $\hat{s}$ and $\hat{p}$ are the normalized Stokes vectors of the optical signal and ASE noise, respectively [1]. Thus, if we neglect the term $\operatorname{DOP}_{n}(\hat{s} \cdot \hat{p})$ for the estimation of $Q$-factor, it will cause an error determined by $10 \log \left(1+\mathrm{DOP}_{n}(\hat{s} \cdot \hat{p})\right)$ in decibel scale. However, it should be noted that this equation provides only the upper limit of the estimation errors if OSNR is very low. The exact analysis is complicated and involves the dependency on OSNR, modulation format, and receiver characteristics, etc. Assuming the Maxwellian pdf of $\mathrm{DOP}_{n}$ and the uniform pdf of $\hat{s} \cdot \hat{p}$, the probability that the estimation error becomes larger than $x \mathrm{~dB}$ can be obtained as

$$
\begin{aligned}
& \text { Probability }\{\mid \text { error } \mid \geq x(\mathrm{~dB})\} \\
& \approx \frac{1}{2}\left\{\operatorname{erfc}\left(\frac{2\left(10^{\frac{x}{10}}-1\right)}{\sqrt{\pi}\left\langle\mathrm{DOP}_{n}\right\rangle}\right)+\operatorname{erfc}\left(\frac{2\left(1-10^{-\frac{x}{10}}\right)}{\sqrt{\pi}\left\langle\mathrm{DOP}_{n}\right\rangle}\right)\right\}
\end{aligned}
$$

for $\left\langle\mathrm{DOP}_{n}\right\rangle \ll 1$ and $x<3$.

\section{EXPERIMENT AND RESULTS}

Fig. 2 shows the experimental setup used to verify the analytical result described above. The outputs of 20 lasers were multiplexed and sent to the transmission link consisted of 15 spans. The first 10 channels (channel number: $1 \sim 10$ ) operated in the range of $1547.3 \sim 1550.9 \mathrm{~nm}$ (channel spacing: $50 \mathrm{GHz}$ ), while the other channels (channel number: $11 \sim 20$ ) operated in the range of $1556.2 \sim 1559.8 \mathrm{~nm}$. Each span was made of an erbium-doped fiber amplifier (EDFA), an optical attenuator, a polarization controller, and a PDL element. The span loss was set to be $10 \mathrm{~dB}$ by using an optical attenuator in every span. We set the optical power of each channel to be $-10 \mathrm{dBm}$ at the input of EDFA. The PDL of each span was set to be about $0.57 \mathrm{~dB}$ by using the PDL element. The polarization controllers were used to randomize the polarization states of optical signals. We filtered the ASE noise at $1553.8 \mathrm{~nm}$ (where there was no signal) by using two optical bandpass filters in cascade (bandwidth: $0.3 \mathrm{~nm}$ ). Thus, we could measure the DOP of ASE noise without the influence of optical signals. The cascaded filter had a negligible PDL $(<0.1 \mathrm{~dB})$ at the center wavelength. The total PMD of the transmission link was measured to be about $1.6 \mathrm{ps}$. As a result, we could neglect the DOP variation of ASE noise caused by PMD. We measured the DOP of the filtered ASE noise 500 times by using a polarization analyzer, while randomly varying the polarization controllers. Fig. 3(a) shows the DOP distributions of the ASE noise measured after transmission of 15 EDFA spans. The measured data agreed well with the calculated Maxwellian curve by using (7) and (8). However, when the total PDL of the link (i.e., PDL/span $\times(\text { number of spans })^{1 / 2}$ ) is larger than $2 \mathrm{~dB}$, the total noise could be increased significantly (i.e., $\left|\vec{P}_{n}^{\text {out }}\right|+D_{n}^{\text {out }}>n$ ), which, in turn, violates our 


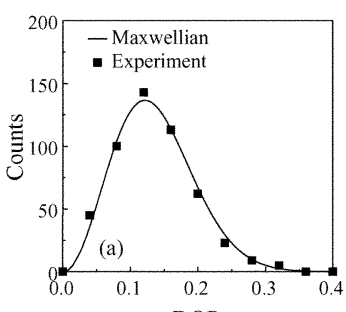

DOP

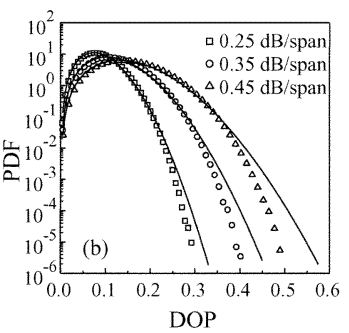

Fig. 3. (a) DOP distributions of the ASE noise measured after the transmission of 15 EDFA spans in comparison with the Maxwellian curve obtained by using (7) and (8). (b) PDF of the DOP of ASE noise obtained by using Monte Carlos simulation (symbols) in comparison with the calculated Maxwellian curves (solid lines). The number of amplifier spans was 30 .
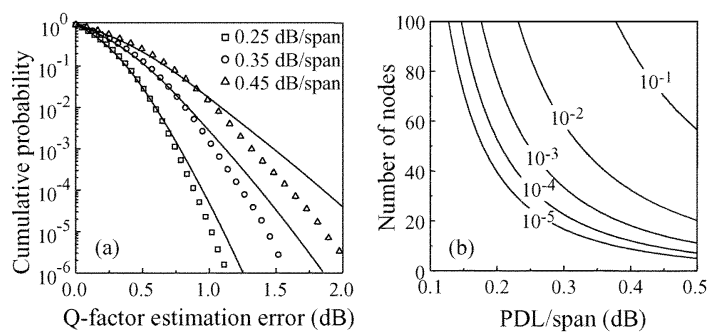

Fig. 4. (a) Cumulative probabilities of the errors in the estimated $Q$-factors by using OSNR (caused by the partially polarized ASE noise due to PDL) in a transmission link consisted of 30 amplifier spans. The symbols are the simulated data and the solid curves are the calculated values. (b) The probability that the error in the estimated $Q$-factor by using OSNR (caused by the partially polarized ASE noise due to PDL) becomes larger than $1 \mathrm{~dB}$.

approximation used in (3) [4]. As a result, the DOP distribution could deviate from the Maxwellian curve in the tails. To evaluate this deviation, we performed Monte Carlo simulations for a 30 -span transmission link while varying the PDL/span to 0.25 , 0.35 , and $0.45 \mathrm{~dB}$ (thus, the total PDL was varied to $1.37,1.92$, and $2.46 \mathrm{~dB}$, respectively). Fig. 3(b) shows the result in comparison with the calculated curves. This figure shows that the simulated data agree well with the calculated curves when the $\mathrm{PDL} /$ span is smaller than $0.25 \mathrm{~dB}$. However, as the PDL/span was increased, the discrepancies between the simulated and calculated values also increased at the tails. Fig. 4(a) shows the cumulative probability of the errors in the estimated $Q$-factors by using OSNR (due to the partially polarized ASE noise) for the transmission link consisted of 30 EDFA spans. In Fig. 4, the solid line represents the calculated value by using (10), and the symbols represent the data obtained by using Monte Carlo simulations. In this simulation, we obtained the DOP of ASE noise and $\hat{s} \cdot \hat{p}$ by using the setup in Fig. 1, and then evaluated the $Q$-factor by using (9). The result shows that, as expected in Fig. 3(b), there are substantial discrepancies between the simulated and calculated values when the estimation error is larger than $1 \mathrm{~dB}$. However, in the region where the estimation errors are smaller than $1 \mathrm{~dB}$, the theoretically calculated values agree well with the simulated data regardless of the value of PDL/span. The simulation result also indicated that the pdf of $\hat{s} \cdot \hat{p}$ was not uniform but slightly tilted, unlike the assumption used in our calculation. This was because, due to PDL, the polarization states of both signal and ASE noise were forced to rotate slightly toward the direction of the PDL vector. However, this effect did not cause a significant difference between the calculated and simulated values. Fig. 4(b) shows the probability that the error in the estimated $Q$-factor becomes larger than $1 \mathrm{~dB}$. The results show that, if the PDL/span is smaller than $0.2 \mathrm{~dB}$ (which is a typical value for current systems [1]), the effect of the partially polarized ASE noise on the estimation of $Q$-factor using OSNR is very small even for a long-distance transmission system. For example, the probability that the error in the estimated $Q$-factor becomes larger than $1 \mathrm{~dB}$ is smaller than $10^{-5}$ in a 30 -span transmission system, as long as the PDL/span is smaller than $0.2 \mathrm{~dB}$. However, this probability could increase to $10^{-3}$ if the number of spans is increased to 80 . On the other hand, if the PDL/span is smaller than $0.15 \mathrm{~dB}$, this probability would remain at $10^{-5}$ even in the 80 -span system. The results also show that, in metro networks, the $Q$-factor can be estimated accurately without considering the effect of the partially polarized ASE noise even when the PDL/span is as high as $0.5 \mathrm{~dB}$. However, it should still be noted that this error probability could substantially increase in some extreme cases such as a transoceanic system. Thus, in such cases, the estimated $Q$-factor using OSNR must be corrected properly by measuring the DOP of ASE noise.

\section{SUMMARY}

We have investigated the effect of the partially polarized ASE noise on the estimation of $Q$-factor by using OSNR. For this purpose, we have analyzed the DOP statistics of the partially polarized ASE noise caused by PDL and verified the result experimentally. We then calculated the probability of the errors in the estimated $Q$-factors caused by the partially polarized ASE noise. The results show that, in most cases (i.e., PDL/span < $0.2 \mathrm{~dB}$ ), the $Q$-factor can be estimated with reasonable accuracy by using OSNR even for the long-distance system (number of spans: <40). However, when the transmission link consists of more than 100 amplifier spans, the effect of the partially polarized ASE noise cannot be neglected unless the PDL/span is smaller than $0.15 \mathrm{~dB}$.

\section{REFERENCES}

[1] I. T. Lima, A. O. Lima, Y. Sun, H. Jiao, J. Zweck, C. R. Menyuk, and G. M. Carter, "A receiver model for optical fiber communication systems with arbitrarily polarized noise," J. Lightw. Technol., vol. 23, no. 3, pp. 1478-1490, Mar. 2005.

[2] Y. Sun, I. T. Lima, A. O. Lima, H. Jiao, J. Zweck, L. Yan, G. M. Carter, and C. R. Menyuk, "System performance variations due to partially polarized noise in a receiver," IEEE Photon. Technol. Lett., vol. 15, no. 11, pp. 1648-1650, Nov. 2003.

[3] Y. Sun, A. O. Lima, I. T. Lima, J. Zweck, L. Yan, C. R. Menyuk, and G M. Carter, "Statistics of the system performance in a scrambled recirculating loop with PDL and PDG," IEEE Photon. Technol. Lett., vol. 15, no. 11 , pp. 1648-1650, Nov. 2003.

[4] A. Meccozzi and M. Shtaif, "Signal-to-noise-ratio degradation caused by polarization-dependent loss and the effect of dynamic gain equalization," J. Lightw. Technol., vol. 22, no. 8, pp. 1856-1871, Aug. 2004.

[5] - "The statistics of polarization-dependent loss in optical communication systems," IEEE Photon. Technol. Lett., vol. 14, no. 3, pp. 313-315, Mar. 2002

[6] F. Curti, B. Daino, G. De Marchis, and F. Matera, "Statistical treatment of the evolution of the principal states of polarization in single-mode fibers," J. Lightw. Technol., vol. 8, no. 8, pp. 1162-1165, Aug. 1990. 